\title{
Modelo didáctico analógico como mediador de enseñanza y aprendizaje universitario del Sistema Cardiovascular
}

\author{
Fany Gisela Arrese (iD \\ Departamento de Física, Facultad de Ciencias Exactas y Naturales, Universidad Nacional de La Pampa. \\ Santa Rosa.Argentina.fanyarrese@gmail.com
}

Jorge Luis Olivares iD

Departamento de Biología, Facultad de Ciencias Exactas y Naturales, Universidad Nacional de La Pampa. Santa Rosa.Argentina.olivares.jorgeluis57@gmail.com

MarinaVillarreal

Departamento de Biología, Facultad de Ciencias Exactas y Naturales, Universidad Nacional de La Pampa. Santa Rosa.Argentina.marvillarreal@cpenet.com.ar

Natalia Gisela Vincet (iD

Docente del Ministerio de Educacoón de La Pampa. Santa Rosa.Argentina.giselavincet@yahoo.com.ar

Valeria Alfageme iD

Departamento de Biologia, Facultad de Ciencias Exactas y Naturales, Universidad Nacional de La Pampa.Santa Rosa.Argentina.vale.alfageme@gmail.com

[Recibido: 28 Diciembre 2019. Revisado: 11 Marzo 2020. Aceptado: 24 Mayo 2020]

Resumen: El aula es un espacio donde el docente puede poner al estudiante en el rol de un científico a través del empleo de modelos para predecir y explicar nuevos fenómenos del conocimiento (Bahamonde y Galindo 2016). Esto pone en palabras que los alumnos son constructores de su conocimiento y que aprender Ciencias significativamente implica recrear las teorías en sistemas de representación internas donde los conceptos están relacionados, y que a partir de la investigación acción pueden ser identificados (Gentiletti 2012). El objetivo de esta investigación es analizar los modelos mentales iniciales de los estudiantes, y compararlos con los modelos mentales finales que elaboran luego de varias intervenciones didácticas de corte socioconstructivista. Se realiza un estudio de tipo cualitativo y cuantitativo donde se estudió el proceso de aprendizaje autorregulado y guiado por el docente sobre estructura y función del Sistema Cardiovascular en 57 estudiantes universitarios de la Carrera del Profesorado en Ciencias Biológicas (UNLPam, Argentina). Se analizaron retrospectivamente producciones obtenidas a lo largo de los años, a partir de una consigna y del empleo del Modelo didáctico analógico (MDA) (Galagovsky y Adúriz-Bravo 2001). Se solicitó al estudiante que realizase un esquema del Sistema Cardiovascular con sus conocimientos previos (modelo mental inicial) y que luego de modificaciones personales; de trabajo colaborativo entre pares y la intervención docente con enfoque pedagógico en la interdisciplina efectuara un esquema final individual (modelo mental final). Se encontró que del esquema inicial al final se incrementó significativamente el número de conocimientos o ideas claves es decir, la diferencia de efecto medio fue - 2,01 a favor de los modelos mentales finales ( $\mathrm{t}=-12,05 ; \mathrm{p}<0.01$; g.l. 1). La investigación acción presentada es innovadora en la enseñanza universitaria porque evidencia que pos intervención pedagógica los estudiantes reconfiguraron significativamente los modelos mentales iniciales con una mirada positiva a las Ciencias en el tratamiento pedagógico del Sistema cardiovascular.

Palabras clave: Modelo didáctico analógico; interdisciplina; formación del profesorado; modelos mentales; sistema cardiovascular.

Analog didactic model as a mediator of university teaching and learning of the Cardiovascular System

Abstract: The classroom is a space where the teacher can put the student in the role of a scientist through the use of models to predict and explain new phenomena of knowledge (Bahamonde and Galindo 2016). This puts into words that students are builders of their knowledge and that learning science significantly implies recreating 
theories in internal representation systems where concepts are related, and that from action research can be identified (Gentiletti 2012). The objective of this investigation is to analyze the initial mental models of the students, and compare them with the final mental models that they elaborate after various didactic interventions of a socio-constructivist nature.

A qualitative and quantitative study was carried out where the self-regulated and teacher-guided learning process on the structure and function of the Cardiovascular System was studied in 57 university students of the Biological Sciences Teaching Career (UNLPam, Argentina). Productions obtained over the years were analyzed retrospectively, based on a slogan and the use of the Analogue Teaching Model (MDA) (Galagovsky and AdúrizBravo 2001). The student was asked to make a scheme of the Cardiovascular System with his previous knowledge (initial mental model) and after personal modifications; Collaborative work between peers and the teaching intervention with a pedagogical focus in the interdiscipline will carry out an individual final scheme (final mental model). It was found that from the initial scheme to the end the number of knowledge or key ideas increased significantly, that is, the difference in the mean effect was - 2.01 in favor of the final mental models $(t=$ $-12.05 ; \mathrm{p}<0.01 ; \mathrm{gl} 1)$. The action research presented is innovative in university education because it shows that after pedagogical intervention, students significantly reconfigured the initial mental models with a positive view of the Sciences in the pedagogical treatment of the cardiovascular system.

Keywords: analog didactic model; interdisciplinary; teacher training; mental models; cardiovascular system.

Para citar este artículo: Arrese F.G., Olivares J.L., Villarreal M., Vincet G. y Alfageme V. (2020) Modelo didáctico analógico como mediador de enseñanza y aprendizaje universitario del Sistema Cardiovascular. Revista Eureka sobre Enseñanza y Divulgación de las Ciencias 17(3), 3601. doi: 10.25267/Rev_Eureka_ensen_divulg_cienc.2020.v17.i3.3601

\section{Introducción}

Esta investigación se genera tras una exhaustiva revisión bibliográfica en la cual no se encontraron experiencias a nivel universitario, y con mirada pedagógica desde las interdisciplinas sobre el empleo del modelo didáctico analógico (MDA) para identificar las representaciones generadas por estudiantes sobre estructura y función del Sistema Cardiovascular.

Los estudios acerca de la importancia de conocer las ideas previas de los estudiantes para obtener un aprendizaje relevante han sido una base importante para la enseñanza y aprendizaje socioconstructivista (Coll y Sanchez 2008; Gentiletti 2012). Los científicos modelizan para predecir y explicar nuevos fenómenos, y se espera que los alumnos sean conscientes que los procesos llevados adelante para modelizar la ciencia que aprenden sigue, en esencia, los mismos mecanismos (Bahamonde y Galindo 2016). La perspectiva de modelización busca construir modelos didácticos que permitan trabajar con las ideas cotidianas de los estudiantes y provocar una ampliación hacia modelos más cercanos a las ideas científicas.

La noción de modelos y modelización en contexto áulico tiene diversos significados, según Oliva (2019) la diferencia reside según donde se ponga el foco de estudio, "si el foco se sitúa en los modelos en sí mismos, en los procesos que lo acompañan, en las demandas que requiere de los estudiantes, en los recursos instrumentales que permiten representarlos o en las estrategias que regulan en conjunto cada una de esas posibilidades" (Oliva 2019). En este artículo se exploran los modelos mentales iniciales y los que logran al final de su formación estudiantes del Profesorado en Ciencias Biológicas. Es decir, considera la modelización como la progresión de los modelos (Oliva 2019). La enseñanza planteada por la cátedra de cuerpo humano II perseguirá la evolución de los modelos mentales de los estudiantes mediante el desarrollo de un taller teórico práctico con diversas actividades que tensionen los modelos mentales iniciales de los estudiantes. Así, luego de cada una de las actividades del taller, el estudiante debe enriquecer el esquema con el que comunica sus ideas de sistema cardiovascular. 
Se evaluaron conocimientos sobre estructura y función del Sistema Cardiovascular a partir de los modelos mentales iniciales y los elaborados después de la intervención docente mediante el MDA (Galagovsky y Adúriz-Bravo 2001). Los MDA fueron empleados como mediadores de la enseñanza y aprendizaje en el aula como línea de formación curricular en las Asignaturas de Cuerpo Humano del Profesorado en Ciencias Biológicas.

\section{Marco teórico}

Sabemos que no aprehendemos el mundo directamente, sino que lo hacemos a partir de las representaciones que construimos de éste en nuestra mente. El conocimiento científico será representado, y recreado internamente por quien lo comprende de una forma que no necesariamente copia las expresiones lingüísticas de sus principios (Moreira, Greca y Palmero 2002). Por esta razón, consideramos que los alumnos son constructores de su conocimiento y que aprender Ciencias significativamente implica recrear las teorías en sistemas de representación internas donde los conceptos están relacionados y que la investigación acción los pone de manifiesto (Gentiletti 2012; Pruzzo 2013). Para que el estudiante logre nuevos conocimientos y su aplicación, es importante el valor de su motivación académica extrínseca (Ventura, Cattoni y Borgobello 2017; Donolo, Chiecher, Paoloni y Rinaudo 2018). En esta motivación, el docente a partir de la intensión pedagógica manifiesta debe generar un ambiente cognitivo y emocional adecuado, a partir de la escucha de saberes previos, historias académicas que obstruyan el aprendizaje, poner en la esfera de la formación la autocrítica de conocimientos erróneos para confrontarlos con los adecuados como una nueva oportunidad pedagógica para la enseñanza y el aprendizaje (Anijovich y Gonzalez 2011; Daura 2014). Diversas investigaciones demuestran que el aprendizaje autorregulado del estudiante es favorecido por el aprendizaje grupal o socializador (Ventura, Cattonni y Borgobello 2017). El ambiente educativo debe favorecer emociones positivas, porque junto con el acompañamiento docente, estimula a pensar y a que el estudiante construya activamente aprendizajes de nivel superior (Mellado, Borrachero, Brígid, Mel, Dávil, Cañada, Conde, Costillo, Cubero, Esteban, Martínez, Ruiz, Sánchez, Garritz, Mellado, Vázquez, Jiménez, Bermejo 2014; Zohar 2006; Olivares, Arrese, Villarreal, Di Franco, Lozano, Alfageme, Vincent, Damm, Carassay 2019).

\section{Modelos mentales}

"Los modelos mentales son análogos estructurales del mundo; su estructura, y no su aspecto, corresponde a la estructura de la situación que representan" (Moreira et al. 2002). De esta manera, se puede interpretar que un modelo científico es la mediación entre el sistema formal teórico y su interpretación (Adúriz Bravo 1999; Viau, Moro, Zamorano, Gibbs 2008).

Los modelos mentales están formados por elementos y relaciones que representan de forma análoga un fenómeno (Greca y Moreira 1998), en nuestro caso de sistema cardiovascular. Todos los elementos y relaciones presentes en el modelo mental deben tener significado o función. Es ésta característica la que permite enriquecerlos y modificarlos y pasar de modelos mentales iniciales, con ciertos elementos y relaciones, a modelos mentales más elaborados. Es a través del proceso de enseñanza y aprendizaje del sistema cardiovascular que podemos acercar el modelo mental de los estudiantes al modelo científico de sistema cardiovascular.

La imagen visual es una representación analógica, es decir, son representaciones mentales a las que se recurre para recuperar o captar la esencia de los fenómenos, o al menos, lo que el estudiante ha considerado relevante. Esto no supone necesariamente la construcción de un modelo mental explicativo y predictivo que las sustente, sin embargo, es la propiedad del modelo mental de ser análogo-estructural la que nos permite inferir las relaciones no explicitadas entre sus componentes. Cada elemento del modelo mental y cada relación debe 
tener un papel simbólico (Rodríguez Palmero, Marrero Acosta y Moreira 2001; Greca y Moreira 1998). Por ejemplo, si un alumno construye un modelo mental sobre la circulación de la sangre en el cuerpo, ese modelo debe tener algún elemento, alguna entidad mental, que represente los vasos del cuerpo, el sentido en que circula la sangre y la concentración de gases que ésta tiene, porque con estos elementos se genera un sustituto. Además, deben existir relaciones causales que le permiten operar con el modelo y hacer previsiones respecto al funcionamiento.

Las personas operamos con modelos mentales, el profesor enseña con modelos mentales y los alumnos aprenden con modelos mentales. Cuando los estudiantes exteriorizan su modelo mental sobre el sistema cardiovascular, lo hacen apoyándose en representaciones externas, entendidas éstas como la expresión concreta de un modelo mental en algún registro semiótico determinado (lenguaje natural, imagen, maqueta, esquema) (Buckley, Gobert, Kindfield, Horwit, Tinker, Gerlits, Wilensky, Dede y Willet 2004). Es decir, los docentes podemos establecer cierto grado de correspondencia entre el modelo conceptual (el esquema o dibujo del estudiante) y su modelo mental de la situación, que permiten inferir cuáles de las cuatro ideas científicas del modelo científico de sistema cardiovascular el estudiante está comunicando.

En resumen, el modelo científico de sistema cardiovascular está formado por cuatro ideas claves, el corazón como bomba; presiones diferenciales; concentración de gases y hematosis. Los modelos mentales de los estudiantes, inferidos a partir de sus dibujos o esquemas, pueden comunicar algunas o todas las ideas. Es objeto de esta investigación conocer cuáles son esas ideas presentes en los estudiantes.

\section{Implicación de los modelos mentales en el aprendizaje relevante. Modelización}

Cuando el docente enseña los modelos científicos espera que el alumno pueda elaborar modelos mentales que le permitan dar significado (Rodríguez Palmero et al. 2001). Es a partir de interactuar con los modelos científicos que los modelos mentales de los estudiantes se van modificando hasta encontrar una configuración que los satisfaga. Naturalmente, cuando el alumno llega a las clases de cuerpo humano II ya tiene modelos mentales construidos por la enseñanza de sistema cardiovascular recibida en el secundario u en otras cátedras a los que denominaremos modelos mentales iniciales. Cabe la posibilidad de que los modelos mentales que traen al aula los estudiantes puedan estar incompletos, incluyan conceptos erróneos o contradictorios, pero son funcionales y precursores de sistemas mentales más estables, los cuales deben ser considerados para arribar a la obtención de aprendizajes significativos. Estos conocimientos cotidianos y elaborados en su historia de aprendizaje es importante conocerlos porque son los que cuentan antes de iniciar el abordaje del tema en la Asignatura. Para construir un MDA es necesario "conocer profundamente el tema que se quiere enseñar, abstraer sus conceptos nucleares y las relaciones funcionales entre dichos conceptos y traducir todo a una situación, lo más inteligible posible para el alumnado. Estos MDA, provienen de la vida cotidiana, de la ciencia ficción o del sentido común" (Galagovsky y Adúriz-Bravo 2001). En este trabajo se estudian los modelos mentales iniciales y los finales elaborados por estudiantes universitarios tras varias intervenciones didácticas.

\section{Objetivos}

- Identificar las ideas presentes en los modelos mentales del Sistema Cardiovascular a partir del análisis de los esquemas elaborados por los estudiantes. 
- Comparar los modelos mentales iniciales y finales luego de varias intervenciones didácticas de corte socioconstructivista.

- Evaluar si el MDA es una estrategia que favorece la progresión de los modelos mentales iniciales de los estudiantes.

\section{Metodología}

Este estudio retrospectivo abarca más de 10 años de trabajo educativo, en el cual los MDA fueron empleados como mediadores de la enseñanza y aprendizaje en el aula (Galagovsky y Adúriz-Bravo 2001). Para trabajar con los MDA y con el proceso de modelización en la enseñanza de las ciencias, es necesario que la estrategia didáctica opere en tres momentos. Un primer momento está orientado a trabajar con la explicitación de las ideas previas que tienen los estudiantes sobre el tema y es fundamental que se deje un registro escrito sobre estas ideas, que puede ser un esquema, una red conceptual, un relato, un cuestionario. En nuestro caso será la elaboración de un esquema que represente el conocimiento anatomofisiológico del Sistema Cardiovascular.

En un segundo momento, se presenta la información proveniente de la ciencia erudita. Esta puede estar presentada a través de textos o exposición del docente, en nuestro caso está dada por la exposición del docente y que transcurre a lo largo de varias clases. El trabajo de los estudiantes es, al finalizar cada clase, reelaborar sus esquemas iniciales, encontrando en ellos contenidos erróneos o incompletos (Ventura et al. 2017). En esta instancia se hace una comparación entre la nueva información proveniente del modelo científico, el modelo mental que el estudiante tiene y el que obtiene por aprendizaje colaborativo con sus pares (Gentiletti 2012). De esta forma incorpora nuevos elementos y nuevas relaciones para reconfigurar su representación mental (Barquero 1995; Rodríguez Palmero et al. 2001). Finalmente, el tercer momento es el de la metacognición, este momento requiere que el alumno reflexione sobre los progresos en sus esquemas, los recorte, los simplifique y les otorgue rangos de validez conceptual y operacional, entre otros. Por lo cual definiremos como modelos mentales finales a aquellos que los estudiantes representen luego de las intervenciones didácticas donde el docente agrega elementos y relaciones con la intención de ampliar, complementar y reestructurar los modelos mentales iniciales que los estudiantes traían al aula. Entendemos como modelos mentales finales, a aquellos con los cuales el estudiante finaliza la Cursada de la Unidad.

Se analizaron las producciones de 57 estudiantes universitarios del Profesorado en Ciencias Biológicas. Dado que la cátedra Cuerpo Humano II tiene alrededor de 4 o 5 estudiantes por año se analizaron todas las producciones. Cada producción cuenta con un mínimo de dos esquemas y un máximo de cuatro. Esta diversidad en las producciones se debe a que se analizó la misma consigna desde el año 2003 al 2018, es decir, a través de los años se fue incrementando las instancias de esquematizar y re-esquematizar el sistema cardiovascular y por ello existen más registros de reflexión sobre los conceptos involucrados en sistema cardiovascular.

La estrategia de enseñanza y aprendizaje estuvo enmarcada en un Taller, teórico y práctico, en donde se alternaron instancias de trabajo individual o cooperativo. La actividad personal tuvo como objetivo que los estudiantes hicieran un aprendizaje autorregulado (Ventura et al. 2017).

En la primer instancia, luego de que el docente indique los objetivos y el proceso de enseñanza que va tener el Taller, le solicita al estudiante realizar un esquema en la cual ponga en evidencia conceptos de anatomía (estructura del corazón), fisiología (función del corazón y los vasos) con el apoyo disciplinar de la Química y la Física. Esta actividad implica que los estudiantes 
realicen un esquema donde indiquen sus conceptos académicos previos sobre las cavidades cardíacas y la entrada y salida de vasos, indicando con distintos colores y flechas los sentidos, la concentración de gases y diferencia de presiones entre los vasos a partir de su salida de los ventrículos derecho e izquierdo.

Posteriormente en el desarrollo del taller, el docente realiza distintas intervenciones orales con la intención de poner a prueba los modelos mentales iniciales de los estudiantes. Mediante aportes teóricos acompañados de imágenes se habilita un diálogo bidireccional y participativo para que el estudiante vaya contrastando sus aciertos y errores en el esquema inicial.

En la segunda clase los estudiantes reciben aporte teórico, en esta oportunidad sobre el sistema vascular periférico al corazón, sus distintos vasos, calibres de los mismos, estructura diferencial entre venas y arterias que representan la relación con la función de presión en los vasos etc. Se propone a los estudiantes reflexionar acerca de qué sucede en las arterias cuando las personas presentan presión arterial elevada, y para ello se presentan situaciones problemas. Se dialoga sobre antecedentes o casos presentados en la familia, que tipo de tratamiento realizan etc., es decir, motivar a los estudiantes a explicar qué aspectos del sistema cardiovascular, alimentación u otros factores puedan producir alteración en la presión arterial con el objetivo de trabajar sobre la prevención de Enfermedades Cardiovasculares. En el práctico el docente enseña a los estudiantes a tomar la presión arterial y posteriormente invita a los que saben a que se la tomen a los demás, y al finalizar los estudiantes conversan sobre los valores de presión arterial normal y elevada. Con los aportes recibidos los estudiantes deben volver a reformular en forma individual el esquema que realizaron al inicio del taller.

En la tercera actividad el docente reúne los esquemas realizados y los distribuye al azar (procurando que no toque el propio). Reunidos de a dos y bajo el acompañamiento del docente, los estudiantes opinan y clasifican la información obtenida de los esquemas de sus compañeros (Gentiletti 2012). La cuarta actividad, es el esquema final del sistema cardiovascular, el cual se produce en el parcial. En esta instancia se considera que el esquema inicial ha sido enriquecido en sucesivas ocasiones con el aporte de las actividades del taller.

El docente finaliza el taller cuando al hacer la devolución de los resultados de los parciales indica cuáles son las ideas válidas del modelo científico de cardiovascular. A decir de Monereo (2007) el aprendizaje se aplica a un contexto áulico en cual el docente emplea imágenes con diferencia de colores para distinguir cuál es la sangre oxigenada de la carbooxigenada, se utilizan esquemas y materiales como tensiómetro aneroide para contextualizar los conocimientos en un ambiente donde primen emociones positivas para lograr una aplicación social del conocimiento como la prevención del aumento de la presión arterial o hipertensión arterial (Monereo 2007; Olivares et al. 2019).

\section{Análisis de datos}

La evaluación de los esquemas consistió en observar si los estudiantes comunican gráficamente las cuatro ideas claves que conforman el modelo científico del Sistema Cardiovascular al cual se espera que arriben. En los esquemas finales se espera observar que el estudiante describa contenidos de disciplinas como Biología, Física y Química con el enfoque de interdisciplina de la asignatura. Estas cuatro ideas claves que permiten caracterizar y comparar la evolución de los modelos mentales de los estudiantes son: el corazón como bomba; presiones diferenciales; concentración de gases y hematosis.

1. El corazón como bomba: esta idea está compuesta por dos conceptos a) que el corazón está compuesto por aurículas y ventrículos y b) que posee válvulas cardíacas. Se deben comunicar ambos conceptos para que la idea clave, el corazón como bomba, 
esté completa. Estos conceptos se pueden inferir a través de la presencia de un primer grupo de signos utilizados con la intención de representar las válvulas presentes y el corazón con sus ventrículos y aurículas. En el esquema se observa un corazón dividido en cuatro compartimientos con signos como AI (aurícula izquierda), AD (aurícula derecha), VI (ventrículo izquierdo), VD (ventrículo derecho), o palabras como ventrículos, aurículas, izquierdo, derecho. Las válvulas aparecen identificadas como discontinuidades en las líneas que separan las aurículas de los ventrículos y en ocasiones se acompaña con una referencia que indica el nombre de la válvula.

2. Presiones diferenciales: aquí también es necesario que estén presentes dos conceptos para que la idea clave esté completa. Se debe representar a) que al corazón llegan venas y salen arterias y b) que la sangre tiene una sola dirección de circulación con diferentes presiones. Estos conceptos se pueden inferir a partir del uso de signos con la intención de representar los diferentes vasos como las venas y las arterias a través de utilizar esquemas similares a un tubo, la identificación de algunos vasos indicado con palabras cómo "vena", "arteria" o con el nombre específico de alguna de ellas y la dirección de circulación de la sangre en el interior del corazón a través del uso de flechas que entran al corazón desde las venas, pasan de aurículas a ventrículos y salen por las arterias.

3. Concentración de gases: se puede inferir a partir del uso de signos con la intención de representar la concentración de gases, en los esquemas al utilizar el color azul (sangre carbo oxigenada) o rojo (sangre oxigenada). En ocasiones también se incluye el color de las aurículas y ventrículos del corazón.

4. Hematosis: se puede inferir a partir del uso de signos con la intención de representar las cuatro venas pulmonares, que provienen de los dos pulmones ( 2 venas de cada uno) que al producir el intercambio gaseoso son identificadas de color rojo y que llegan a la aurícula izquierda. En algunos esquemas estas cuatro venas pulmonares están representada por cuatro tubos, o por cuatro círculos o por la salida de un tubo principal de la aurícula izquierda con el número cuatro al lado y/o la leyenda de "venas pulmonares".

Para establecer un criterio que homogenice las cantidades de producciones de los estudiantes, se decidió establecer el primer dibujo como el modelo mental inicial, es decir, aquel dibujo que los estudiantes realizan antes de que se desarrolle el tema, a partir de sus saberes previos y modelo mental final al último dibujo realizado por el estudiante para la instancia de evaluación final de la unidad, es decir, aquel que fue elaborado luego de al menos tres intervenciones docentes para desarrollar el tema.

El análisis de los datos se realiza en dos niveles. Comienza con el nivel de análisis I, en donde se realiza una descripción de los signos (flechas, palabras conectoras, colores y notas entre otros) encontrados en los esquemas como un acercamiento a las ideas que forman el modelo científico del Sistema Cardiovascular. En el Nivel II, se compara y analiza estadísticamente el número de ideas claves presentes en los esquemas iniciales y en los esquemas finales para evaluar el aprendizaje en proceso. Se utilizó la técnica estadística de comparación de medias a través de la prueba t para muestras relacionadas (distribución t-student) con un nivel de significancia de $0,05(5 \%)$. Todos los datos fueron procesados empleando el programa estadístico STATISTICA versión 13.5.0.17 (Sampieri 2018; Johnson y Kuby 2008). 


\section{Resultados}

Al estudiar la modificación de los modelos mentales de los 57 estudiantes encontramos dos barreras para su análisis. La primera barrera fue de tipo metodológica, al igual que lo referido por Loarces, Ferrer y García (2019) al dilema asociado a encontrar las regularidades en las ideas que los estudiantes quieren comunicar. La segunda dificultad encontrada fue instrumental y tiene que ver con los símbolos a través de los cuales los estudiantes comunican las ideas. Para la primera dificultad, se analizaron los esquemas con la intención de identificar y clasificar las ideas centrales del modelo científico del Sistema Cardiovascular que comunican los estudiantes. Con respecto a la segunda dificultad, se buscó asociar símbolos y signos que representaran esas ideas. Una vez establecido estos acuerdos metodológicos, el desafío fue asociar el modelo mental inicial, con las ideas y relaciones, que tenían los estudiantes y luego contrastarlo con las ideas y relaciones que tenían en un modelo mental final, que sería aquel que pudieron comunicar luego del proceso de enseñanza (Galagovsky y Adúriz-Bravo 2001).

\section{Nivel de análisis I}

Este nivel de análisis se centra, en la identificación de los signos, que son los sustitutos de las ideas que forman los modelos mentales que tienen los estudiantes. A partir de la identificación de los signos, por inferencia, se asocian las ideas científicas del modelo de sistema cardiovascular a las ideas que tienen los estudiantes en sus modelos mentales. En esta instancia se trabajó con 114 esquemas generados por 57 estudiantes y se establecieron cuatro ideas claves que permiten caracterizar la evolución de los modelos mentales generados por los estudiantes.

En la Figura 1 se muestran como ejemplo los esquemas realizados por dos estudiantes sobre las ideas claves que forman el modelo científico del Sistema Cardiovascular propuesto por la asignatura. Los esquemas muestran lo que el estudiante representa con sus ideas previas a la izquierda al que denominamos modelo mental inicial y a la derecha luego de elaborar y reelaborarlos a partir del proceso de aprendizaje en el desarrollo de la temática al que denominaremos modelo mental final por ser elaborado luego del proceso de enseñanza.

La inclusión de nuevos símbolos en los esquemas finales indica que los estudiantes encuentran sustitutos gráficos para comunicar las nuevas relaciones y conceptos del modelo mental que tienen sobre el sistema cardiovascular. Significa, que los modelos mentales de los estudiantes fueron modificados o al menos se puede asegurar que se incorporaron nuevos elementos y relaciones a sus modelos mentales iniciales de Sistema Cardiovascular. 


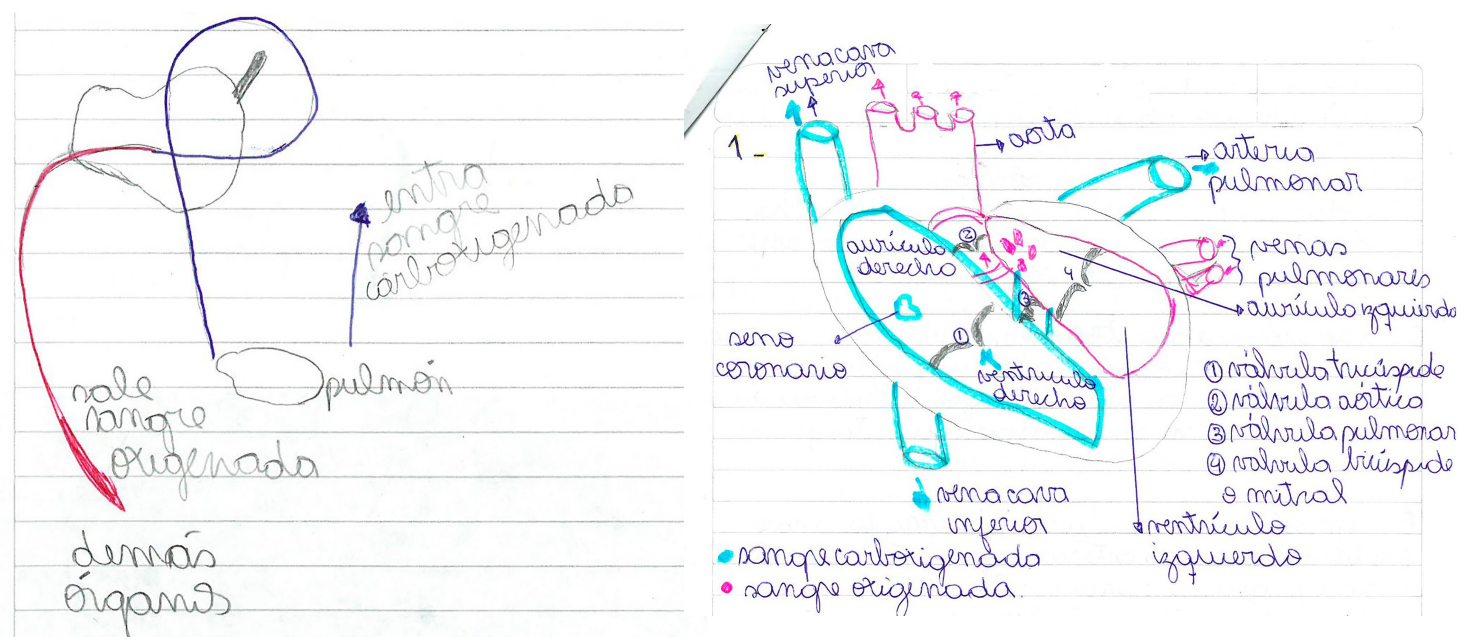

Alumno A. Ideas del modelo mental inicial

Alumno A. Ideas del modelo mental final

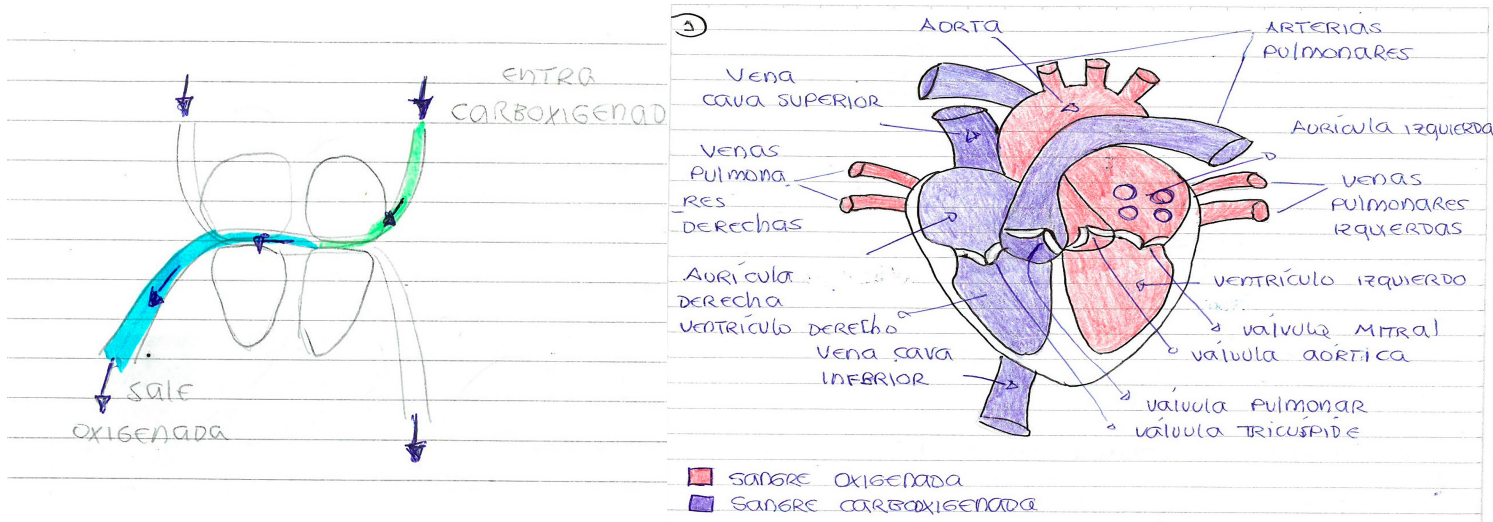

Alumno B. Ideas del modelo mental inicial

Alumno B. Ideas del modelo mental final

Figura 1. Comparación de modelos mentales iniciales y finales sobre el modelo científico de Sistema Cardiovascular en esquemas realizados por dos estudiantes.

\section{Nivel de análisis II}

A continuación, se describe y comparan las ideas comunicadas por los estudiantes en los esquemas iniciales y los esquemas finales con respecto a la idea nuclear.

1. Corazón como bomba: inicialmente se ve que el 31\% de los estudiantes logra comunicarla ya que, para construir esta idea clave es necesario comunicar tanto la presencia de válvulas cardíacas como la identificación de aurículas y ventrículos. Esta idea en el esquema final es triplicada ( $88 \%$ ) en el número de alumnos que pueden incluir símbolos para comunicarla.

2. Presiones diferenciales: se encuentra en los esquemas iniciales que el $2 \%$ de los estudiantes indica símbolos que representan la dirección de circulación de la sangre, aumentando al 60\% en los esquemas finales de la idea central de presiones parciales.

3. Concentración de gases: se observa que en los esquemas iniciales el 44\% de los estudiantes logra la representación de colores para identificar y diferenciar la sangre 
oxigenada y carboxigenada. Este porcentaje se ve duplicado $(82 \%)$ en la comunicación de los esquemas finales.

4. Hematosis: es la idea clave que aparece con menor frecuencia en los esquemas iniciales ya que, sólo un 3\% de los estudiantes puede comunicarla, aunque esta idea clave tubo el mayor incremento en la comunicación debido a que el porcentaje en los esquemas finales alcanzó el 56\%.

En la Tabla 1 se muestran los resultados comparados obtenidos a partir del análisis de las cuatro ideas claves de Sistema Cardiovascular trabajadas durante el proceso de enseñanza y aprendizaje sobre la temática. Se muestra cuáles de las cuatro ideas claves del modelo científico están presentes en los modelos mentales iniciales y cuáles de las cuatro ideas claves conforman los modelos mentales finales de los estudiantes. $\mathrm{Al}$ analizar el proceso evolutivo de las ideas, se observa que en todas hubo un incremento relativo, destacándose la idea sobre las presiones diferenciales en los vasos, la cual engloba un concepto anatómico como es la identificación de arterias y venas; y uno funcional como es la dirección de la corriente de la sangre que se identifica con la presión hidrostática en los vasos.

Tabla 1. Ideas claves del modelo científico de sistema cardiovascular comunicadas por los estudiantes en los esquemas iniciales y finales.

\begin{tabular}{lccc}
\hline \multicolumn{1}{c}{ Ideas clave } & $\begin{array}{c}\text { Esquema inicial } \\
\mathbf{n}=\mathbf{5}\end{array}$ & $\begin{array}{c}\text { Esquema final } \\
\mathbf{n}=\mathbf{5 7}\end{array}$ & $\begin{array}{c}\text { Ideas } \\
\text { comunicadas en } \\
\text { comunicadas en } \\
\text { el modelo inicial final }\end{array}$ \\
\hline a. Grafica aurículas y ventrículos & $27(47 \%)$ & $54(95 \%)$ & - \\
b. Grafica válvulas cardíacas & $18(31 \%)$ & $50(88 \%)$ & - \\
El corazón como bomba (idea & $31 \%$ & $88 \%$ & Está comunicada
\end{tabular}

clave completa formada por ambas variables)

\section{Presiones diferenciales en los vasos}

a. Grafica venas/arterias
b. Señaliza la dirección de la
circulación de la sangre

Presiones diferenciales en los vasos (idea completa formada por ambas variables)

Diferencia color rojo/azul en los vasos

Grafica 4 venas pulmonares de color rojo desembocando en la aurícula izquierda

$\begin{array}{lccc}17(30 \%) & 50(88 \%) & - & - \\ 1(2 \%) & 34(60 \%) & - & - \\ 2 \% & 60 \% & \begin{array}{c}\text { No está } \\ \text { comunicada }\end{array} & \text { Está comunicada }\end{array}$

3. Concentración de gases

$25(44 \%) \quad 47(82 \%) \quad$ Está comunicada Está comunicada

4. Hematosis

$2(3 \%) \quad 32(56 \%) \quad$ No está $\quad$ Está comunicada

4 Ideas claves del modelo científico de sistema cardiovascular: 1. corazón como bomba, 2. Presiones diferenciales de los vasos, 3. Concentración de gases, 4. Hematosis. Se considera que uno o dos estudiantes de los 57 no son suficiente para decir que la idea está comunicada. 
Este nivel de análisis tuvo el propósito de comparar los modelos mentales iniciales y finales de los estudiantes y analizar si hubo un cambio significativo. Para ello recordemos que, el modelo científico de Sistema Cardiovascular está formado por cuatro ideas claves y que los esquemas que realizan los estudiantes para exteriorizar sus modelos mentales (iniciales y finales) no necesariamente comunican las cuatro ideas, algunos pueden tener una o más de las ideas claves. En fin, La intención es comparar los modelos mentales para determinar si hay diferencia significativa entre el modelo mental inicial y el modelo mental final.

En los modelos mentales iniciales la idea que se comunica con mayor frecuencia $44 \%$ es la diferencia de colores entre la sangre oxigenada y carbooxigenada seguido por la idea del corazón como bomba con el 31\%. Si bien, el 47\% de los estudiantes entiende que el corazón está formado por aurículas y ventrículos, no logra identificar las válvulas cardíacas y por eso varios estudiantes no pueden construir una idea completa acerca de que el corazón funciona como bomba. La idea que aparece con menos fuerza $2 \%$ es la idea de presiones diferenciales, es decir, logran distinguir con mayor facilidad las venas y las arterias, pero no así la dirección de circulación de la sangre. Por lo tanto, podemos afirmar que en los modelos mentales iniciales, menos de la mitad (44\% y 31\%) de los estudiantes logran comunicar dos de las cuatro ideas claves.

En los modelos mentales finales, la idea que se comunica con mayor frecuencia (88\%) es que el corazón funciona como bomba, ya que los estudiantes son capaces de identificar las aurículas y ventrículos y las válvulas cardíacas. Lo que realmente sorprende, es el incremento de estudiantes que logran comunicar la idea como hematosis que pasa de un $3 \%$ en los modelos iniciales a un 56\% en los finales como así también la idea de presiones diferenciales.

Esto supone que los modelos mentales de los estudiantes fueron modificados, lo cual se confirmó con el análisis estadístico realizado sobre la diferencia de medias para datos apareados. El test t-student para datos apareados arroja una diferencia estadísticamente significativa entre el número de ideas claves del modelo mental inicial y del final de los estudiantes, es decir, la diferencia de efecto medio fue - 2,01 a favor de los modelos mentales finales $(\mathrm{t}=-12,05 ; \mathrm{p}<0.01 ;$ g.l.1).

\section{Discusión}

Espontáneamente, las personas construyen modelos mentales para dar significado al mundo que los rodea, estos modelos son funcionales según los intereses personales (Moreira et al. 2002). La educación debe generar algo más que brindar conocimientos al estudiante, debe hacer de esos modelos mentales personales, modelos que tengan un mayor rango de validez, y que se acerquen a los modelos científicos que se estudian. Por ello, si la finalidad de la enseñanza es que los alumnos construyan modelos mentales que permitan acercarse al conocimiento científico, es necesario que el docente explicite el trabajo de modelización y le dedique el tiempo necesario a modelizar y aplicarlos a situaciones reales (Moreira et al. 2002; Tamayo Alzate y Sanmartí 2003).

Presentamos una investigación acción retrospectiva en la cual el objetivo fue identificar los modelos mentales iniciales y los finales sobre estructura y función del Sistema Cardiovascular elaborados por estudiantes universitarios de la carrera del Profesorado en Ciencias Biológicas tras varias intervenciones didácticas de corte socioconstructivista (Daura 2017). Para generar contextos de enseñanza y aprendizaje se diseñaron estrategias como la enseñanza de la medición de la presión arterial con tensiómetros anareoides, lo cual daba lugar a un espacio de diálogo entre docente y estudiante intentando generar un mayor número de emociones positivas hacia la estrategia propuesta (Monereo, 2007; Mellado et al. 2014; Olivares et al. 2019). 
Encontramos que un mínimo del $56 \%$ a un máximo del $88 \%$ de los estudiantes logra comunicar las cuatro ideas claves del Sistema Cardiovascular en sus modelos mentales finales. Se puede concluir que las ideas claves construidas en los modelos mentales finales de los estudiantes son más numerosas que en los modelos mentales iniciales ya que sus esquemas finales contienen más signos y símbolos. En base a esto estamos en condiciones de afirmar que mediante el aprendizaje en proceso y autorregulado, aquellos estudiantes que pudieron comunicar a través de sus esquemas tres o cuatro ideas centrales de sistema cardiovascular, tienen una mayor comprensión del tema. Este resultado se debería a que los estudiantes en su modelo mental asignan mayor relevancia a la información científica y logran encontrar los sustitutos para comunicar sus ideas (Rodríguez Palmero et al. 2001; Greca y Moreira 1998; Loarces et al. 2019). Al comparar los modelos mentales iniciales y los modelos mentales finales de sistema cardiovascular, se puede ver claramente que estos modelos mentales progresan en varios aspectos dando el estudiante al empleo del gráfico un papel importante en este proceso metacognitivo, como lo mencionan otros investigadores (Alarcón, Alcas, Alarcón, Natividad y Rodríguez 2019).

La investigación acción indica el progreso significativo en la adquisición del nivel de conocimientos de los estudiantes favorecido por el aprendizaje autorregulado y en el cual el docente dirige con la teoría y la repetida generación de esquemas a la meta propuesta que es la aplicación de conocimientos de Biología, Física y Química para trabajar en el aula sobre la prevención de Enfermedades Cardiovasculares (Pruzzo 2013; Pintrich y De Groot 1990). Esto explicaría que el mayor progreso de los estudiantes fue en la idea sobre las presiones diferenciales en los vasos, la cual engloba un concepto anatómico como es la identificación de arterias y venas; y uno funcional como es la dirección de la corriente de la sangre que se identifica con la presión hidrostática en los vasos. Esto podría deberse a que el docente trabaja en contexto con situaciones problemas como es la Hipertensión arterial, que es una de las causas más frecuentes de enfermedades cardiovasculares.

Consideramos que los diferentes avances en los modelos mentales de los estudiantes se debe al aprendizaje autorregulado, en el cual el docente acompaña en el proceso con una enseñanza dirigida al tema de Sistema Cardiovascular pero depende de aspectos personales de cada estudiante (Daura et al. 2017). Aquellos estudiantes que sólo logran obtener conceptos sueltos de Sistema Cardiovascular elaboran un modelo mental que asigna menor relevancia y significado a la misma información, mostrando dificultades para interpretarla en el propio modelo, y esto manifiesta que su modelo mental es más simple, poco predictivo y explicativo (Rodríguez Palmero et al. 2001; Greca y Moreira 1998). De esta manera se observa que algunos estudiantes reestructuran más que otros, comprenden más, encuentran mayores contradicciones y asignan y reasignan nuevos significados a los elementos y relaciones de sus modelos mentales a situaciones de enseñanza planteadas. No existen evidencias sobre el empleo del MDA desde las interdisciplinas para evaluar conocimientos sobre estructura y función del Sistema Cardiovascular en la enseñanza universitaria. A nivel primario, Galavosky y Edelsztein (2018) investigaron errores del aprendizaje del Sistema Cardiovascular en estudiantes de 10 a 12 años, a partir de un cuestionario utilizando algunas variables de estudio similares a las investigadas por nuestro grupo como es el significado del color de la sangre, aunque, en nuestro protocolo los errores fueron detectados en esquemas y evaluados en procesos en estudiantes universitarios de la carrera de Biología. Las investigadoras arriban por este método a detectar los aprendizajes erróneos sobre este Sistema el cual denominan Obstáculos Epistemológicos de Aprendizaje (OEA). 


\section{Conclusión}

Presentamos un estudio retrospectivo donde se emplea para la investigación acción el MDA y que abarca más de 10 años de tarea pedagógica, en donde se analiza el aprendizaje en proceso de una Unidad didáctica sobre Sistema Cardiovascular. A través de esquemas efectuados por estudiantes universitarios de la Carrera del Profesorado en Ciencias Biológicas se estudian los modelos mentales iniciales o formados por las ideas previas y los finales o construidos posteriormente a la intervención pedagógica.

Los resultados encontrados ponen de manifiesto la necesidad de instaurar un modelo de formación docente activo y participativo en el que debe haber motivación académica en instaurar un proceso de aprendizaje autorregulado (Ventura et al. 2017).

Se demuestra que para lograr las metas en este modelo socioconstructivista, el rol docente debe ser no solo interesar al estudiante desde lo motivacional, sino su compromiso de acompañar, guiar, coordinar y evaluarlo en un espacio socialmente adecuado (Donolo et al. 2008).

La investigación acción permitió identificar nuevos sustitutos en los esquemas mentales realizados por los estudiantes cuando finaliza la unidad de Sistema Cardiovascular. Esto permite inferir que los modelos mentales iniciales se reconfiguraron y, en principio, se amplió el margen en el que son válidos, aceptando la hipótesis que se puede plantear, predecir y explicar lo que se puede hacer con ellos. Esto se explica porque, desde un enfoque sociocognitivo, la creación de modelos mentales para generar aprendizajes significativos se originan desde una propuesta del docente que motiva y guía a la acción, en donde la reiteración y el refuerzo de la actuación pedagógica son fundamentales para que el estudiantado aprenda a autorregular sus aprendizajes (Ventura et al. 2017; Daura 2017).

El empleo del MDA (Galagovsky y Adúriz-Bravo 2001) demuestra ser un mediador de la enseñanza y aprendizaje de contenidos sobre estructura y función sobre Sistema cardiovascular, al comparar entre los modelos mentales iniciales y los finales, demostrando que el número de ideas comunicadas claves comunicadas por los estudiantes pasó de dos a cuatro. Además, el porcentaje de estudiantes que logra comunicar dos ideas en los modelos mentales iniciales fue menos de la mitad (44\% y 31\%), mientras que en los modelos mentales finales el porcentaje de estudiantes que comunicar las cuatro ideas va desde el 56\% al 88\%. Es decir, los estudiantes universitarios reconfiguraron significativamente sus conocimientos, ampliaron el grado de predicción y la explicación acercándose, en mayor o menor medida, al modelo científico (Moreira et al. 2002).

\section{Referencias}

Anijovich, R y González, C. (2011).Evaluar para aprender: conceptos e instrumentos. Buenos Aires, Argentina: Aique.

Adúriz Bravo, A. (1999). Elementos de teoría y de campo para la construcción de un análisis epistemológico de la didáctica de las ciencias. Tesis de Maestría. Universidad Autónoma de Barcelona. España

Alarcón, A., Alcas, N., Alarcón, H., Natividad J., y Rodríguez, A. (2019). Empleo de las estrategias de aprendizaje en la universidad. Un estudio de caso. Propósitos y Representaciones, 7 (1), 10-32.

Bahamonde, N., y Galindo, A. A. G. (2016). Caracterización de modelos de digestión humana a partir de sus representaciones y análisis de su evolución en un grupo de docentes y 
auxiliares académicos. Enseñanza de las ciencias: revista de investigación y experiencias didácticas, 34(1), 129-147.

Barquero, B. (1995). La representación de estados mentales en la comprensión de textos desde el enfoque teórico de los modelos mentales. Tesis Doctoral. Universidad Autónoma de Madrid. España

Buckley, B.,Gobert, J.D., Kindfield, A.C.H., Horwit, P. ,Tinker R., Gerlits, B., Wilensky U., Dede, C. y Willet, J. (2004). Model-based teaching and learning with biologica: what do they learn? How do they learn? How do we know?. Journal of Science Education and Technology. 13, 23-41.

Coll, C y Sánchez, E. (2008). Presentación. El análisis de la interacción alumno-profesor: líneas de investigación. Revista de Educación (346), 15-32.

Daura, F. T. (2014). Los docentes universitarios y su concepción sobre el aprendizaje, la autorregulación y la personalización educativa. Un análisis desde la teoría fundamentada. Forum Qualitative Sozialforschung / Forum: Qualitative Social Research, 15(2), 15-32. https://ri.conicet.gov.ar/handle/11336/92340

Daura, F. T. (2017). Aprendizaje autorregulado e intervenciones docentes en la universidad. Revista Educación, 41(2), 1-18.

Donolo, D., Chiecher, A., Paoloni, P. y Rinaudo, M. C. (2008). MSLQe-MSLQvv motivated strategies learning questionnaire. Propuestas para la medición de la motivación y el uso de estrategias de aprendizaje. Río Cuarto, Argentina: Fundación Universidad Nacional de Río Cuarto.

Galagovsky, L. R., y Adúriz-Bravo, A. (2001). Modelos y analogías en la enseñanza de las ciencias naturales. El concepto de modelo didáctico analógico. Enseñanza de las Ciencias, 19(2), 231-242. https:// dialnet.unirioja.es/servlet/articulo?codigo $=243382$

Galavosky, L.R. y Edelsztein, V.C. (2018). Obstáculos de aprendizaje en niños de 10-12 años sobre el tema sistema circulatorio humano: una propuesta teórica en base a evidencias. Ciência y Educação (Bauru), 24 (2), 283-299.

Gentiletti, M. G., (2012). Construcción colaborativa de conocimientos integrados. Aportes de la psicología cultural en las prácticas de la enseñanza. Contenidos y competencias. Buenos Aires, Argentina: Ediciones novedades educativas.

Greca, I. M., y Moreira, M. A. (1998). Modelos mentales, modelos conceptuales y modelización. Caderno catarinense de ensino de física, 15(2), 107-120.

Jonson, R., y Kuby, P. (2008). Estadistica Elemental: Lo Esencial. Santa Fe, México: Cengage Learning Editores.

Loarces R. G., Ferrer G. F., y García F. G. (2019). Evolución de los modelos mentales sobre fosilización tras el proceso de enseñanza-aprendizaje. Revista Eureka sobre Enseñanza y Divulgación de las Ciencias 16(2), 2102-1-2102-14.

Mellado, V., Borrachero, A.B., Brígido, M., Melo, L.V., Dávila, M.A., Cañada, F., Conde, M.C., Costillo, E., Cubero, J., Esteban, R., Martínez, G., Ruiz, C., Sánchez, J., Garritz, A., Mellado, L., Vázquez, B., Jiménez, R., Bermejo, M.L. (2014) Las emociones en la enseñanza de las ciencias. Enseñanz̧a de las Ciencias, 32 (3), 11-36.

Monereo, C. (2007). Hacia un nuevo paradigma del aprendizaje estratégico: El papel de la mediación social, del self y de las emociones. Electronic Journal of Research in Education Psychology, 5(13), 497-534. 
Moreira, M.A., Greca, I. M., y Palmero, M. L. R. (2002). Modelos mentales y modelos conceptuales en la enseñanza y aprendizaje de las ciencias. Revista brasileira de Pesquisa em Educaşão em Ciências, 2(3), 84-96.

Oliva, J.M (2019) Distintas acepciones para la idea de modelización en la enseñanza de las ciencias. Enseñanza de las ciencias, 37 (2), 5-24.

Olivares, J. L., Arrese, F., Villarreal, M., Di Franco, M. G., Lozano, A., Alfageme, V., Vincet, G.N., Damm, N., Carassay, M.L. (2019). Análisis de emociones referidas por estudiantes universitarios ante distintas estrategias didácticas utilizadas durante su formación profesional. Diálogos Pedagógicos. 4 (34), 83-97.

Pintrich, P. R. y De Groot, E. V. (1990). Motivational and Self-Regulated Learning Components of Classroom Academic Performance. Journal of Educational Psychology, 82(1). 33-40. Doi: 10.1037/0022-0663.82.1.33

Pruzzo, V (2013). Las prácticas del profesorado. Mediadores didácticos para la innovación. Córdoba Argentina: Editorial Brujas

Rodríguez Palmero, M. L., Marrero Acosta, J., y Moreira, M. A. (2001). La Teoría de los Modelos Mentales de Johnson-Laird y sus principios: una aplicación con modelos mentales de célula en estudiantes del Curso de Orientación Universitaria. Investigações em ensino de ciências. Porto Alegre. 6(3) 243-268. https://lume.ufrgs.br/handle/10183/141225

Sampieri, R. H. (2018). Metodología de la investigación: las rutas cuantitativa, cualitativa y mixta. México: McGraw Hill.

Tamayo Alzate, O E, y Sanmartí P, Neus. (2003). Estudio multidimensional de las representaciones mentales de los estudiantes. Aplicación al concepto de respiración. Revista Latinoamericana de Ciencias Sociales, Niñezy Juventud, 1(1), 181-205.

Ventura A C, Cattoni M S y Borgobello A. (2017) Aprendizaje autorregulado en el nivel universitario: Un estudio situado con estudiantes de psicopedagogía de diferentes ciclos académicos. Revista Electrónica Educare , 21(2) 1-20.

Viau, J. E., Moro, L.E., Zamorano, R.O. y Gibbs, H.M. (2008). La transferencia epistemológica de un modelo didáctico analógico. Rev. Eureka Enseñ. Divul. Cien., 5(2), 170-184.

Zohar, A (2006). El pensamiento de orden superior en las clases de ciencias: objetivos, medios y resultados de investigación. Enseñanza de las ciencias, 24(2), 157-172. 